to appear IEEE Conference on Robotics and Automation. May 1989, Scottsdale, AZ.

Technical Report CUCS-363-88

\title{
HAPTIC OBJECT RECOGNITION USING A MULTI-FINGERED DEXTROUS HAND
}

\author{
Peter $K$. Allen \\ Kenneth S. Roberts \\ Department of Computer Science \\ Columbia University \\ New York, N. Y. 10027
}

\begin{abstract}
It is becoming increasingly clear that robotic systems need to have capabilities similar to the human haptic system in order to perform complex grasping, manipulation and object recognition tasks using dextrous hands. This paper is an exploration of using a dextrous, multi-fingered hand for high-level object recognition tasks. The paradigm is model-based recognition in which the objects are modeled and recovered as superquadrics, which are shown to have a number of important attributes that make them well suited for such a task. Experiments have been performed to recover the shape of objects using sparse contact point data from the hand with promising results. We also present our approach to using tactile data in conjunction with the dextrous hand to build a library of grasping and exploration primitives that can be used in recognizing and grasping more complex multi-part objects.
\end{abstract}

\section{INTRODUCTION}

The use of multi-fingered robotic hands has previously focused on the low-level aspects of control $[10,12,18,24,25]$. While such low-level control is a necessary component for an intelligent grasping system, it must also be linked to an understanding of the higher-level strategies and requirements of complicated grasping $[15,20,21,31]$. Our work at Columbia in dextrous manipulation has focused on these higher level tasks and the building of an overall intelligent robotic system that includes dextrous hands, vision sensors, and multiple degree of freedom manipulators. With the advent of dextrous hands such as the Utah/MIT hand [17] intelligent grasping systems have become a reality. Our research goal is in building a comprehensive grasping environment, capable of performing tasks such as locating moving objects and picking them up, manipulating man-made objects such as tools, and recognizing unknown objects. In addition, we hope to build an integrated programming environment that will allow grasping and grasping primitives to be included in an overall robotic control and programming system. It is becoming increasingly clear that robotic systems need to have capabilities similar to the human haptic system in order to perform complex grasping, manipulation and object recognition tasks using dextrous hands.

This paper is an exploration of using a dextrous, multi-fingered hand for high-level object recognition tasks. Object recognition has traditionally been the domain of vision sensor systems. However, these systems suffer from a number of inherent problems, not the least of which is occlusion. Any visual system will be limited at most to a view that obscures all back-facing areas of the object. In robot manipulation tasks, important areas of the work 
environment are occluded by the end-effector itself. This difficulty is especially acute during the act of acquiring a grasp on an object, when the contact areas will be occluded. It is our belief that both visual and haptic sensor systems are needed in a complex robotic system, and the previous research of Allen [2] and Stansfield [28] has explored and quantified this multisensor capability.

In order to fully understand the power of haptics, we have first set out to perform a series of object recognition tasks using touch alone. If we can achieve success in object recognition using the haptic approach, then we will be able to extend our system's power greatly by using both touch and vision. We are motivated by the research of Lederman and Klatzky [19] who quote a 96-99\% success rate in identifying 100 common objects using two-handed, haptic object recognition. They have also identified a number of important exploratory strategies that humans use in performing these tasks that we are transporting to the robotics domain.

The outline of this paper is as follows: Section 2 describes our use of superquadric primitives for model-based haptic object recognition, section 3 describes our shape recovery procedure from sparse contact data, section 4 describes our work in adding tactile sensing capability to a dextrous hand and section 5 discusses methods for more complex shape recovery and extensions of our work to grasping.

\section{OBJECT MODELS FOR HAPTIC RECOGNITION}

An intelligent robotic system will necessarily have to deal with a combination of intemal models and data gathered from various noisy sensors. Thus, to function in its dynamic, threedimensional environment, an intelligent robotic system must be able to quickly and accurately build these models by combining noisy sensory data. The model-based approach has proven very useful in a variety of recognition problems ranging from image understanding [9] to intelligent wouch sensing $[2,13,28]$. The choice of model for an intelligent haptic recognition system is an important one, since the model chosen must meet the criteria set forth by Mar [23] and others. In essence, the desirable qualities that the model must possess include accessibility, efficiency, stability, uniqueness and scope. In addition, we seek models that are in some sense prototypical or generic, since the manipulation tasks we foresee will be using sets of objects with similar atributes, although differing somewhat in size and shape (tools, man-made objects, etc.). Finally, the modeling system should provide the ability to synthesize shapes as well as providing a convenient paradigm for recovering shape from sensory data. General purpose robotic tasks require that we recover models, or pars thereof, from sensory data. Thus, we seek to extend CAD models in a way that allows efficient recovery from sensory data.

We have chosen to model objects as superquadrics $[3,5,26]$. Some representative models are pictured in Figure 1, and the equation for a superquadric is shown in Figure 2. These models are derived from a parameterization that allows a wide degree of freedom in modeling objects. The parameter space is continuous and allows a smooth change from a cuboid to a sphere to a cylinder, with more complex shapes derivable with the addition of bending and tapering parameters. These "lumps of clay" are deformable by the usual linear stretching and scaling operations and can be combined using boolean set operations to create more complex objects.

In addition to possessing most of the desirable modeling criteria discussed above, the models are surprisingly easy to recover from sparse and noisy sensor data. The small number of parameters needed to recover the shape describes a very large class of objects, that can be equated with generic or prototypical recognition. Efficient recovery of superellipsoids (a 


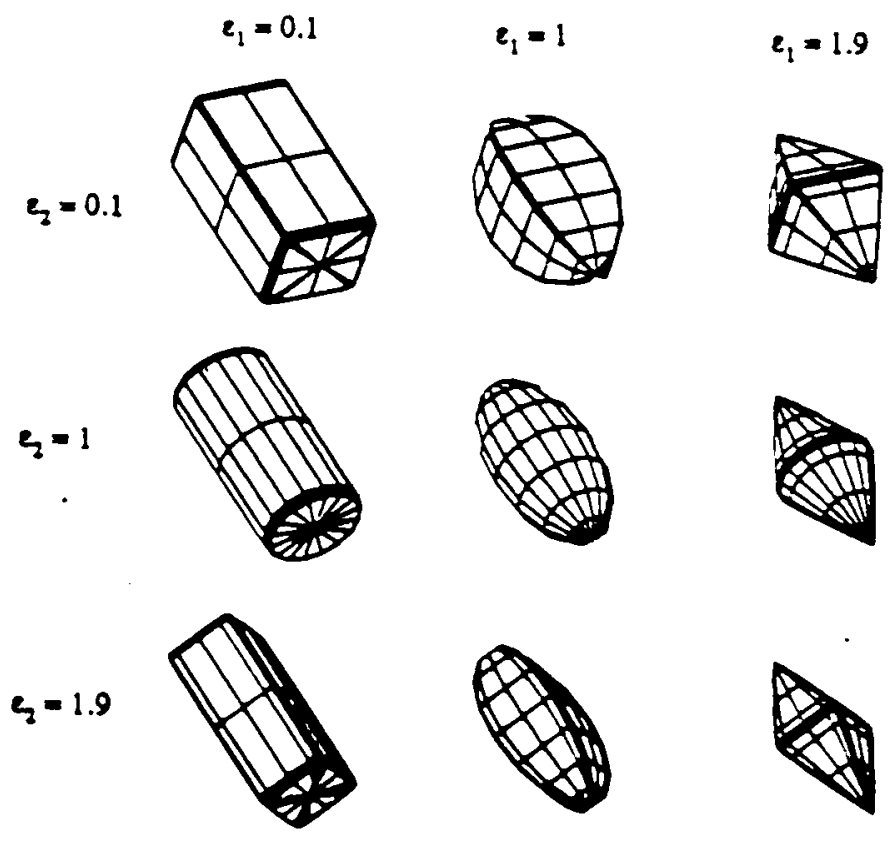

Figure 1: Superquadric shapes.

$$
\mathbf{X}(\eta, \omega)=\left[\begin{array}{c}
a_{1} C_{\eta}^{\varepsilon_{1}} C_{\omega}^{\varepsilon_{2}} \\
a_{2} C_{\eta}^{\varepsilon_{1}} S_{\omega}^{\varepsilon_{2}} \\
a_{3} S_{\eta}^{\varepsilon_{1}}
\end{array}\right]
$$

$C_{\eta}, S_{\infty}$ stand for Cosine( $\left.\eta\right)$ and Sine $(\omega)$.

$\varepsilon_{1}, \varepsilon_{2}$ are the superquadric shape parameters.

$a_{1}, a_{2}, a_{3}$ are scaling factors along the $\mathrm{X}, \mathrm{Y}$ and $\mathrm{Z}$ directions.

Figure 2: Superquadric equation. 
subclass of superquadrics) from vision and range data has already been shown in the work of Pentland [26], Solina [27] and Boult and Gross [7] whe have also studied the error properties of the recovery process [14].

What makes superquadrics particularly relevant for haptic recognition is the following:

- The models are volumetric in nature, which maps directly into the psychophysical perception processes suggested by grasping by containment [19].

- The models can be constrained by the volumetric constraint implied by the joint positions on each finger.

- The models can be recovered with sparse amounts of point contact data since oniy a limited number of parameters need to be recovered. There are 5 parameters related to shape (see Figure 2) and 6 related to position and orientation in space. Global deformations (tapering, bending) may add a few more.

- In addition to the use of contact points of fingers on a surface, the surface normals from contacts can be used to describe a dual superquadric which has the same analytical properties as the model itself.

- The analytic nature of the model created from sparse data allows searching strategies in the model space to proceed in a hypothesize and test fashion as suggested by the work of Allen [1] and Stansfield [28].

\section{HAPTIC RECOVERY OF OBJECT SHAPE}

Previous efforts in recovering shape from grasping have focused on pattem recognition methods of discriminating between samples in many dimensional spaces $[8,22,29]$. Our approach is to actually recover a 3-D model from sparse contact data. We have attempted to create robotic analogues of the exploration strategies humans use in object recognition tasks, noting positional contacts during these explorations. Our experimental appararus is depicted in Figure 3. It consists of a UtahMMT dextrous hand mounted on a PUMA 560 manipulator. The hand contains 16 degrees of freedom, allowing it to grasp the object in a containing grasp. The PUMA provides 6 additional degrees of freedom ( 3 translation, 3 rotation) providing an overall system with 22 degrees of freedom.

The first of these strategies we have implemented is grasping by containment. This is an attempt to understand an object's gross contour and volume by effectively molding the hand to the object. With sparse contact point data, it is unclear how much "information content" is included in this measure. The experiments we present in this paper have shown it is a powerful and useful discriminator of objects, and in the examples we will show, is sufficient to allow robust and accurate shape recovery.

\subsection{Recovery Procedure}

For this initial work on recognition, we have used a simplified procedure to gather data points. Our intent is to use integrated tactile sensors (described below) mounted on the finger links to generate contact position data. However, during our initial trials, our tactile sensors were not yet mounted on the hand. Instead, we opted for a method that used the hand's internal joint angle readings and tendon forces to generate Cartesian positions of contact based upon fingertip contact 


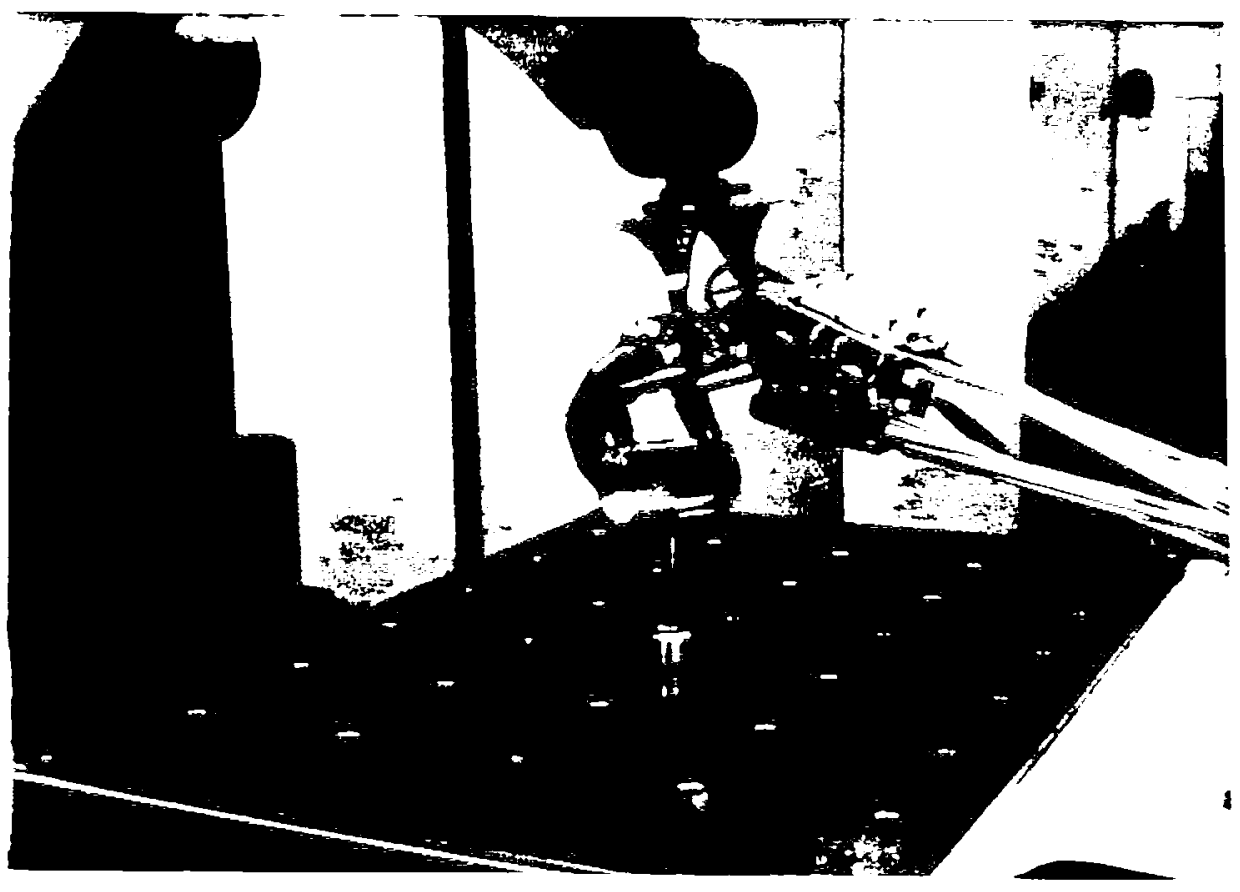

Figure 3: Utah/MIT hand mounted on PL'MA 560

The Puma arm moves the hand to a position in which it will close around the object. The lingers are spread wide during approach. Then the fingers are closed by position commands until the observed force (estimated by the difference between the flexor and extensor indon tensions) exceeds a given threshold. which indicates that the finger is in contact with the object. The joint angle positions are read, and kinematic models of the hand and the Puma arm are used to conver them to $X Y Z$ positions in world coordinates. Then the fingers are opened wide again. and a second containing grasp is executed. with the fingers taking different approach paths. The lingers are spread once again, and the Puma arm moves the hand to the next position.

The sequence of Puma positions is given in advance. Once the contact points are determined using the forward kinematics of the hand derived from the joint angle sensors, the sparse sets of point data are then injected into the recovery algorithm developed by Solina [27]. This algorithm uses a Levenberg-.Marquard non-linear least squares approximation to fit the superquadric "inside-out function". This is an implicit form of the equation in Figure 2 which rccords if a sample data point lies inside. outside or on the surface of the superquadric model. By summing the squared distance of each sample data point from the current model, an error of tit measure is generated that is minimized by the algorithm.

The equation in Figure 2 is for a canonical superquadric located at the origin. Since our sensor data can exist anywhere in the world coordinate space, the algorithm must recover the 6 rotation and translation parameters in addition to the 5 superquadric shape parameters $\left(a_{1}, a_{2}, a_{3}, \varepsilon_{1}, \varepsilon_{2}\right)$. In addition. we allow global deformations to include tapering of superquadric loms. The taper is defined to be a linear tapering with 2 parameters that control the 
tapering in both the $X$ and $Y$ dimensions. The algorithm must recover a minimum of 11 parameters and 13 if the object is tapered.

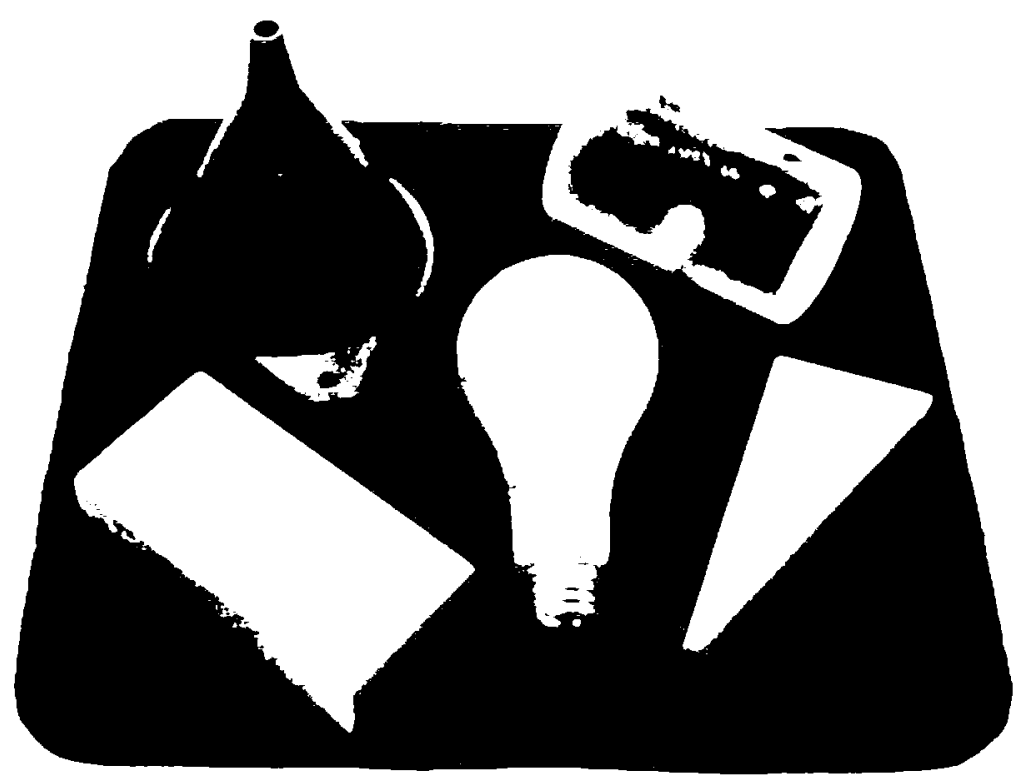

Figure 4: Object Database.

We tested this procedure against a database of 6 objects (shown in figure 4 plus a smaller cylinder). The database included objects that could be modeled as undeformed superquadrics (block. large cylinder, small cylinder) and defomed (tapered) superquadrics (lightbulb. funnel, triangular wedge). The recovered shapes are shown in Figures 5a-e, with the sample data points overlaid on them.

The results of these experiments are quite good, especially considering the sparse nature of the data and the errors in the derived contact points. These errors are a function of the dicuricy and calibration of the robotic arm. the hand joint position sensors and the kinematic model of the hand itself. In spite of this sensor cror. the recovered shapes are an accurate represintation of the actual object's shape. The dala points are overlayed on the recovered shapes to show the closeness of fit and the sparseness of the data. Each object's shape was recovered with extremely sparse anounts of data: typically 30-100 points, depending on the object. It is imporiant to note that this is about two orders of magnitude less than typical range data images which try to recover shape with denser data, that, unlike touch sensing, is limited to a vieupoint that only exposes half the objects surfaces to the sensor. In addition, we are able to calculate in a closed form the volumes of undeformed superquadrics. Volume calculations for the recovered cylinder are within 1-2\% of the actual volume of the object.

We are in the process of revising the recovery algorithm utilizing the ideas of Gross and Boult [14]. In their recovery algonthm, a volume minimization term is not included and the distance metric on the points is measured differenty from the inside-out function used by 
Solina. It should be noted that both recovery schemes work well with denser data (as in range data) but the sparseness of the data points may lead to a modified method that may utilize aspects of both of the recovery methods. We also are including the surface normal constraints formed from the dual superquadric representation into our recovery procedure.

\subsection{Matching}

Once these shapes have been recovered, it is necessary to do object discrimination to correctly identify the objects. The superquadric recovery process supplies 5 relevant parameters that are useful in discriminating among an object's shape and size. The superquadric shape parameters, $\varepsilon_{1}$ and $\varepsilon_{2}$ are good indicators of gross shape and the scaling parameters $a_{1}, a_{2}, a_{3}$ are useful in size discrimination. We have written a simple matching algorithm that matches the recovered superquadric parameters against the database of models, and is able to discriminate among the 6 objects successfully. The algorithm does a simple comparison of the 6 recovered superquadric parameters against each set of model parameters, and takes a weighted sum of these parameter distance measures as the error of fit We experimented with 10 different sets of sampled data, encompassing the 6 objects, some in more than one pose. Using this simple procedure on this limited database, we were able to successfully recognize each object uniquely during each trial.

However, we are not claiming that simple discrimination tests like these are adequate to solve the matching problem. Our intent is to make two important points related to object recognition using sensor data. The first point is that sparse, noisy data can yield good estimates of shape. Second, that this method of haptic shape recovery is viable for a first level of object discrimination in the presence of sparse, noisy data. By effectively pruning a much larger set of models by the procedures outlined above, we can enter the next phase of active sensing with hypotheses about what an object's structure might be, or alternatively, methods to discriminate between objects which all match the sensor data. The recovery algorithm is useful in active sensory feedback loops where initial coarse estimates can be used to derive the shape, position, and orientation of objects to one level of precision and then subsequent active haptic probes can be used to recover the details of the shape to a finer level.

The next steps in this process is to implement other exploratory procedures. including edge following and lateral contour exploration that further discriminate among objects. For these procedures, we will utilize our tactile sensors.

\section{TACTILE SENSING FOR A DEXTROUS HAND}

The UtahMMT hand provides a number of levels of sensory feedback for manipulation. Each joint is provided with a hall effect sensor that can accurately provide positional information on each of the 16 joint angles. In addition, velocity at each joint is computed. The control of the hand is effected through co-contraction of 32 tendons (16 flexion, 16 extension). The force applied to each of these tendons, measured at the wrist of the hand, is also available.

While the level of sensing provided by this hand is better than earlier implemented hands, it still falls far shor of the requirements for a dextrous manipulation system. In particular, what is desired is accurate positional contact information between the hand and a target object, and a measure of the forces exerted by the fingers at these contact points. The sensory feedback provided by the hand does not allow for localization of contacts. Hence, a requirement for the tasks envisioned in this system is a robust and accurate tactile sensor system, utilizing 
sensors mounted on the links of the fingers.

To satisfy this requirement, we are currently implementing tactile sensors on each of the hand's fingers. The technology being used is a piezo-resistive polymeric material manufactured by Interlink, Inc. [16,30] This material has good force response characteristics and is able to be deposited on arbitrary, conformable surfaces. The design of the tactile pads we are using sandwiches the polymer between two pliable sheets of Kapton material that contains electrical etching. The application of forces on the pads provides an increased electrical flow channel between the two sheets as the material within is compressed. Results with this sensor have been good, particularly with respect to signal isolation and hysteresis. We have built an electronics package to multiplex the sensor signals, and hope to achieve a high enough bandwidth in signal processing of the sensors to achieve real-time integration of the tactile data into the grasping procedures. Figures 6 shows the output of tactile responses filtered with median and averaging filters when a pen cap is placed sensor pads. The signal is very localized (the sensing area is on the order of 0.5 inches by 1.0 inches). Figure 7 shows the output of a credit card applied across these sensors, and Figure 8 shows the resulting extracted linear segments from this impression using a Hough transform technique [4].

Using this tactile data, we are in the process of implementing the exploratory procedures of lateral motion on a surface and edge/contour following with the addition of tactile sensing capability to the hand. We will initially implement a number of simple finger level primitives (guarded moves, fingertip force, etc.) for grasping and manipulation and create a library of grasping primitives based upon tactile feedback. These will form the basis for our ability to perform dextrous manipulations.

\section{EXTENDING HAPTIC PERCEPTION}

We wish to extend the exploratory strategies beyond the initial primitives mentioned above. The work of Grimson and Lozano-Perez [13] and Ellis et al. [11] appears to be important here in suggesting new ways to interpret a set of underconstrained data by generating new sensor probes of an environment. This, coupled with matching criteria developed from the model-based appraach suggested above, will allow intelligent high-level reasoning for manipulation tasks. The analytic nature of the proposed superquadric models will allow generation of surface paths and finger constraints that will be central to a particular grasping strategy.

\subsection{Extension to grasping.}

Results from work on haptic exploration and recognition should be very useful for improving performance in grasping an object. After a superquadric has been fit to the object. it can be used both for choosing a grasp from a known set and filling in any parameters required for that grasp; and also for planning a pre-grasp configuration for the hand and an approach path (see [31]). The initial shape recovery process is important in identifying the overall shape of the object in order to perform a preshaping function for the hand as outlined by Iberall [15]. Many features which will be useful for haptic recognition are also good candidates as grasp contact areas on an object: edge, hole, cavity, neck, two-finger opposition. We may expect that many of the local exploratory movements will be useful for adjusting and improving an initial grasp.

The low pass nature of tactile recognition will try to model classes of complex objects into equivalent simpler primitives, particularly with respect to grasping criteria. Given a task of determining what an object to be picked up is and where to grasp it, we can classify the 
object at a coarser level which expands the equivalence class depending upon the task. For example, many objects such as cups, glasses, mugs, etc. can be modeled as a type of cylindrical primitive when the task is simply raising it up to drink. As task level complexity increases (pick up by the handle) then the recovery processes will need to work harder to accomplish the task.

\subsection{Segmentation}

Complex objects are made up of more primitive sub-parts, and a shape recovery procedure will necessarily need to perform a segmentation of a complex object into its sub-parts. As 3-D data is sampled, it has to be associated with a unique surface or volume that it coheres with. Modeling systems by nature are made up of boolean operations on primitives, and discovering which primitive a data sample belongs to is critical. Most systems, in fact, do not do this well. A number of techniques have been used on this problem with varying degrees of success. A common approach is to use regularization techniques that try to smooth the data into a global energy minimum. However, the discontinuities that these systems smooth may, in fact, be the relevant segmentation criteria for forming new model primitives from sensor data.

Our approach to the segmentation problem makes use of the active nature of tactile sensing with controllable fingers. Segmentation in a geometric sense (it may be left to psychology [6] to understand just what "segmentation" is! ) is determined by finding discontinuities on surfaces. Given a set of active fingers that can trace contours and determine face-edge-vertex relationships, it is possible to use the fingers themselves as segmentation devices. The ability to trace a surface and discover significant discontinuities with the appropriate tactile feedback will allow us to recover multiple superquadric volumes that can be combined in a boolean sense to recreate the scene. Multi-finger haptic exploration offers two kinds of help for the segmentation problem. First, because haptic exploration is inherently a multi-stage process. segmentation uncertainties can be resolved by further probes. Second, features which are very helpful for segmentation can be detected by multi-finger strategies. A neck (place where the circumference of an object about an axis comes to a minimum) can be detected by a two-finger opposing or encircling grasp (moved along the axis). A neck is an excellent indicator of nonconvexity, and thus multiple shape primitives. A hole or cavity can be difficult for vision to handle, but is well-suited for haptic perception. It can indicate the presence of a negative shape primitive.

\section{SUMMARY}

We have presented an integrated approach to robotic haptic object recognition that tries to blend the 3-D model and feature based approaches to object recognition with the ability to actively sense objects using both kinaesthetic and tactile feedback. The results for simple objects are quite encouraging and with the advent of more complex exploratory procedures using tactile sensors, we hope to be able to perform recognition on complex multi-part objects using these ideas.

\section{ACKNOWLEDGEMENTS}

This work was supported in part by Dapa contract N00039-84-C-0165, NSF grants DMC-86-05065, DCI-86-08845, CCR-86-12709, IRI-86-57151, Norh American Philips Laboratories, and the AT\&T Foundation. Special thanks to Franc Solina, Terry Boult, and Ari 
Gross for allowing us to use their respective recovery algorithms; Paul Michelman and Peter Armstrong for acquiring the tactile sensor data; and Walter Khan and Bob Stark for building the PUMA hand mount. 


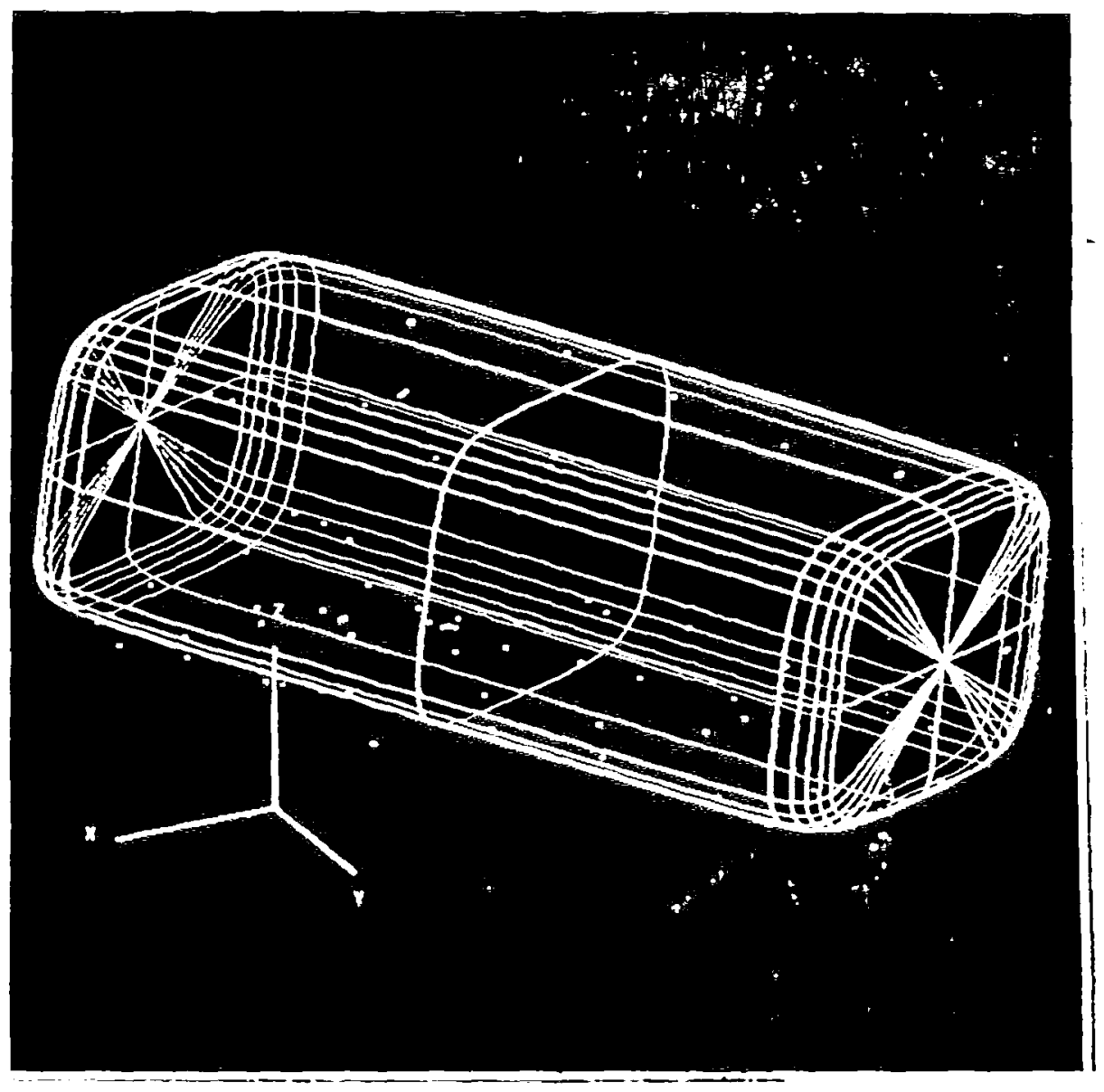

Figure 5a: Recovered shape of a rectangular block. 


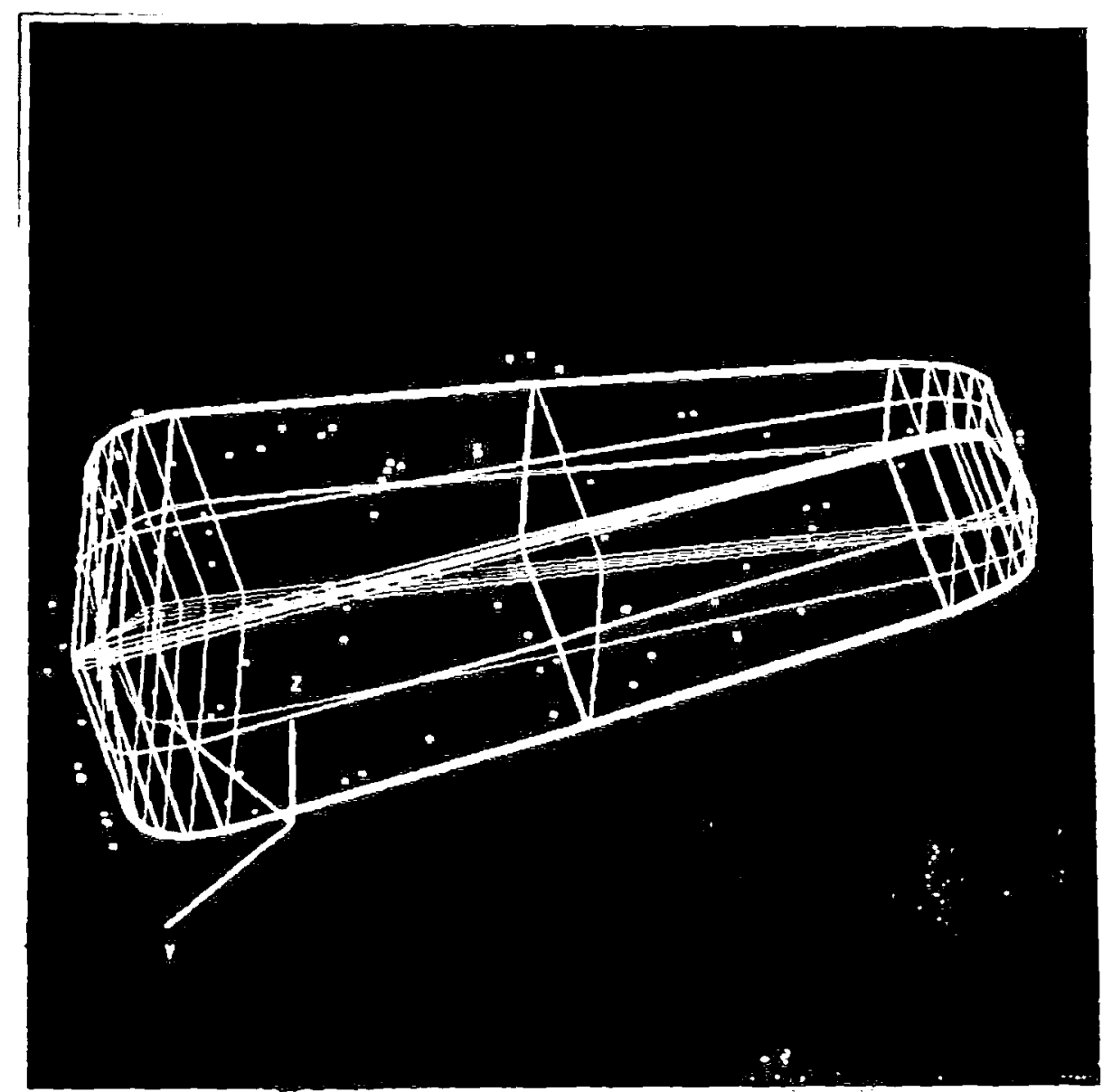

Figure 5b: Recovered shape of a triangular wedge. 


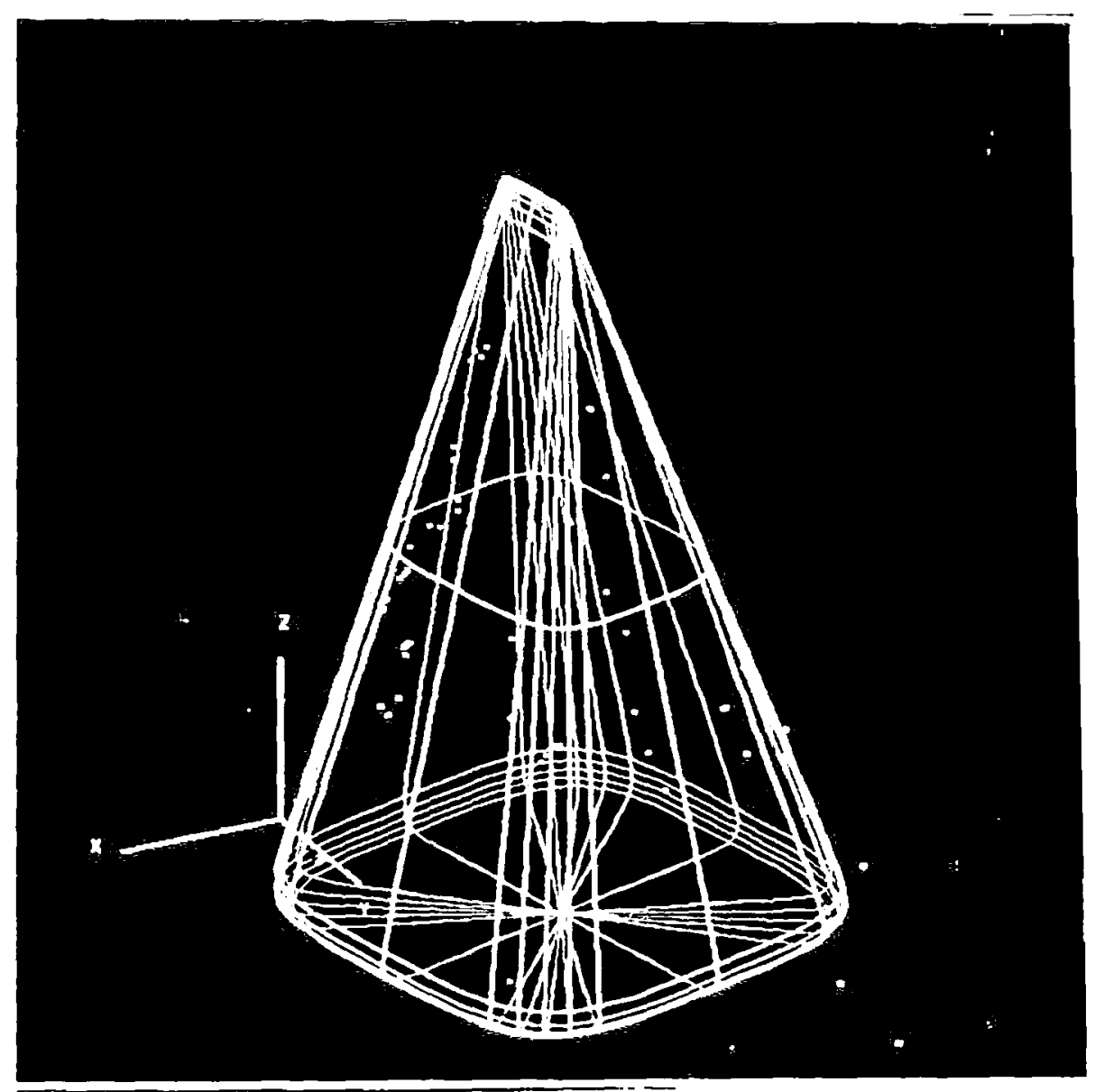

Figure 5c: Recovered shape of a funnel. 


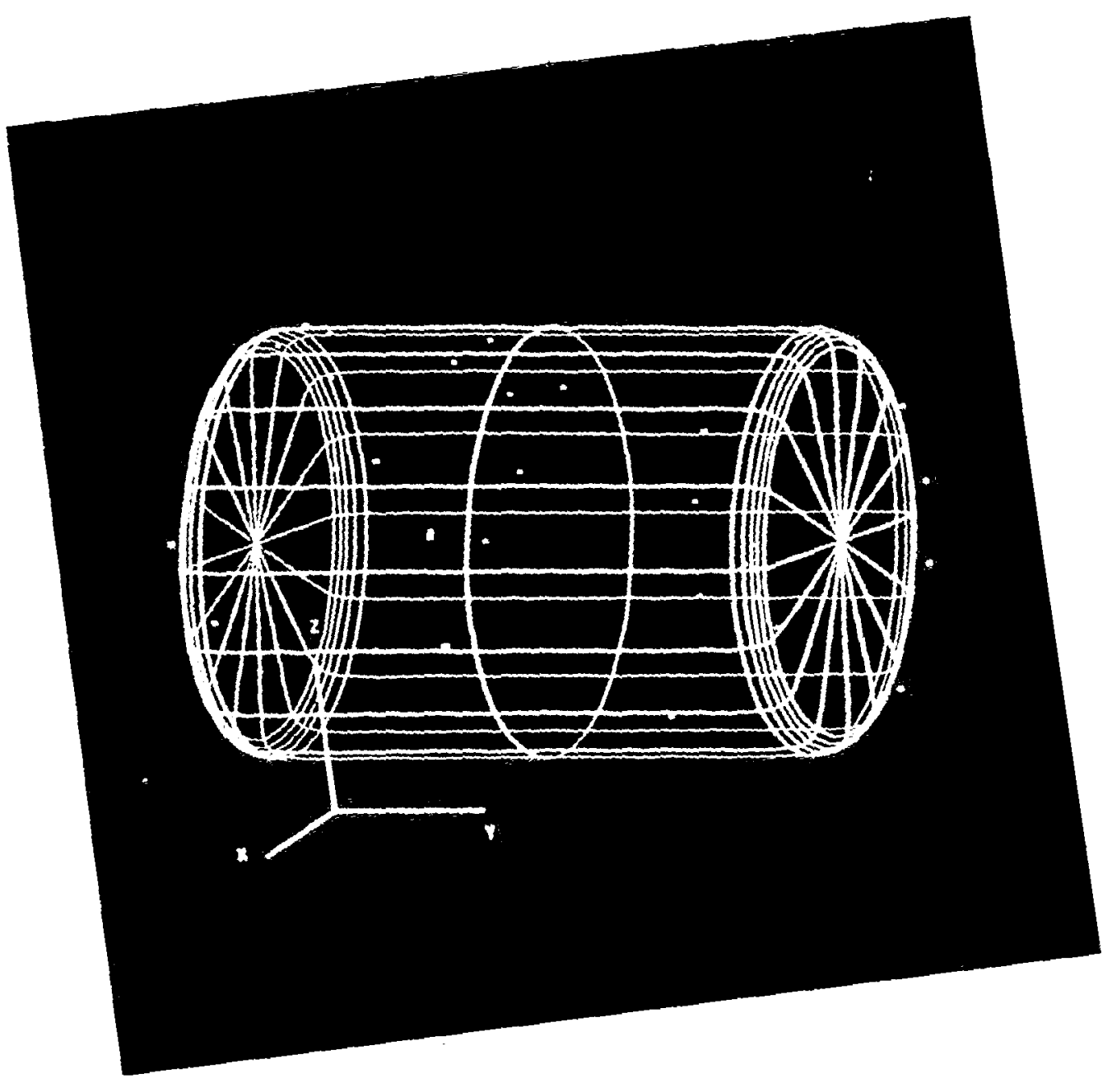

Figure 5d. Recovered shape of a cylinder.

Figure 5d: Recovered shape of a cylinder. 


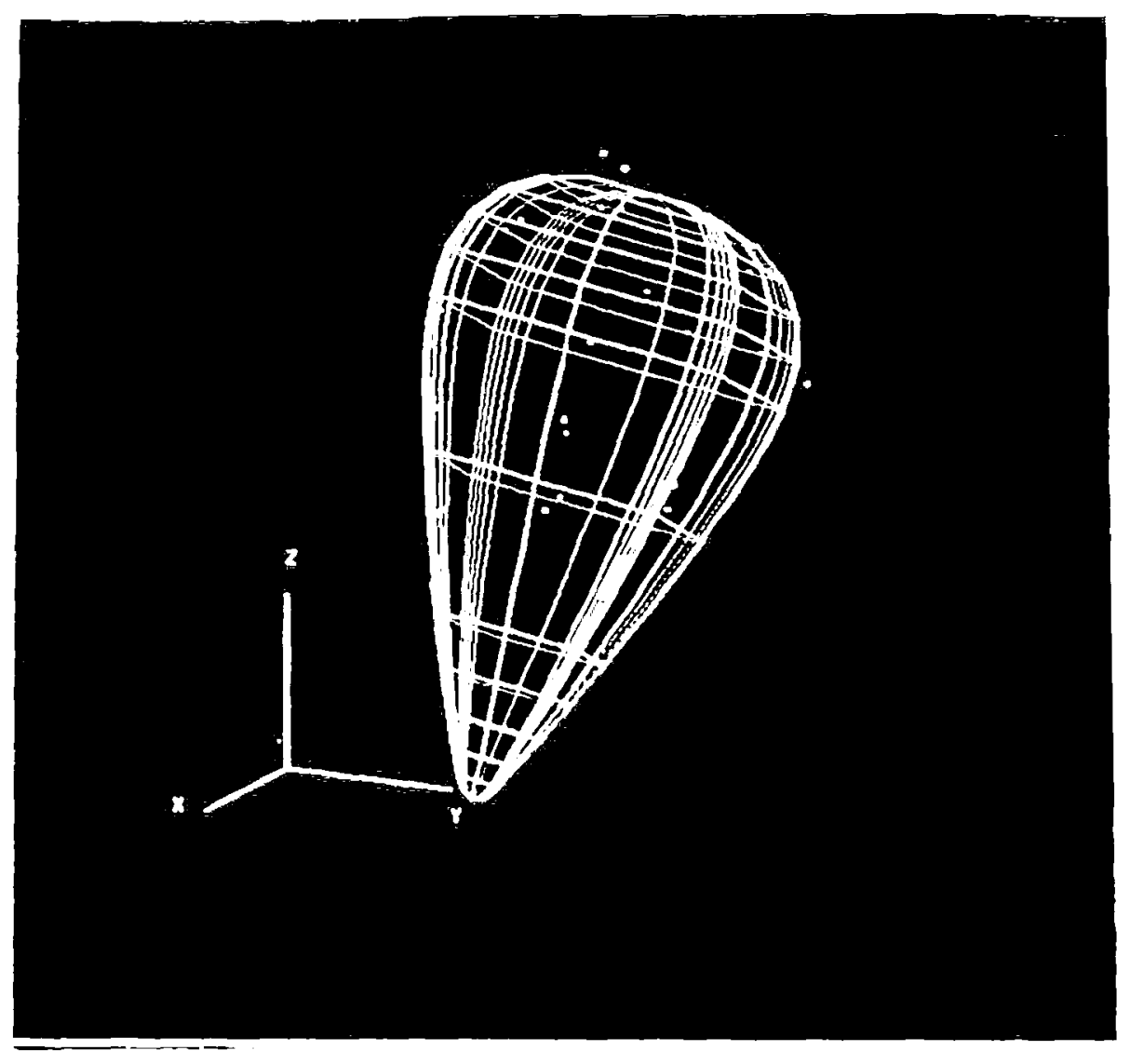

Figure 5e: Recovered shapes of a light bulb. 


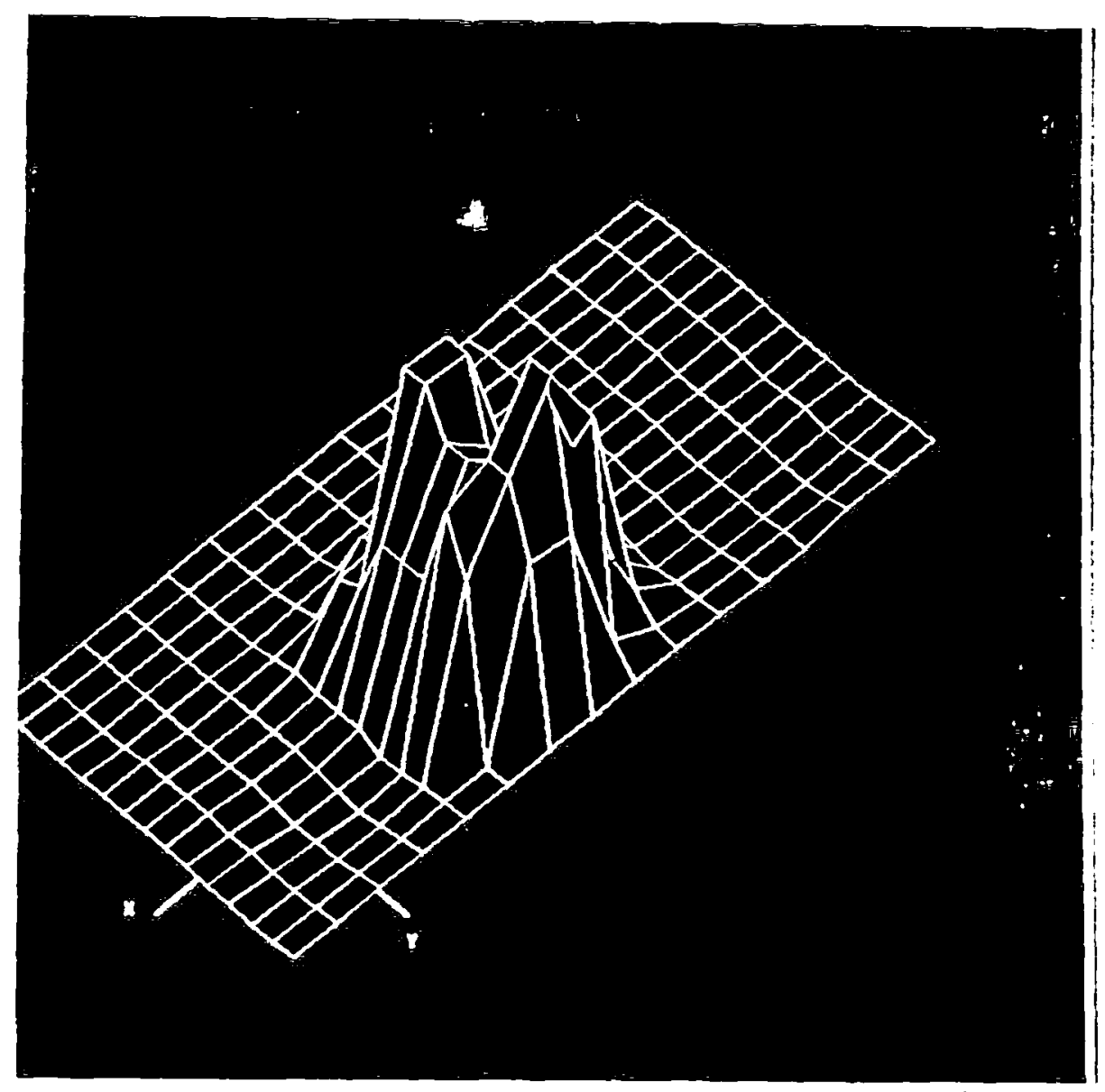

Figure 6: Filtered tactile responses for a pen cap. 


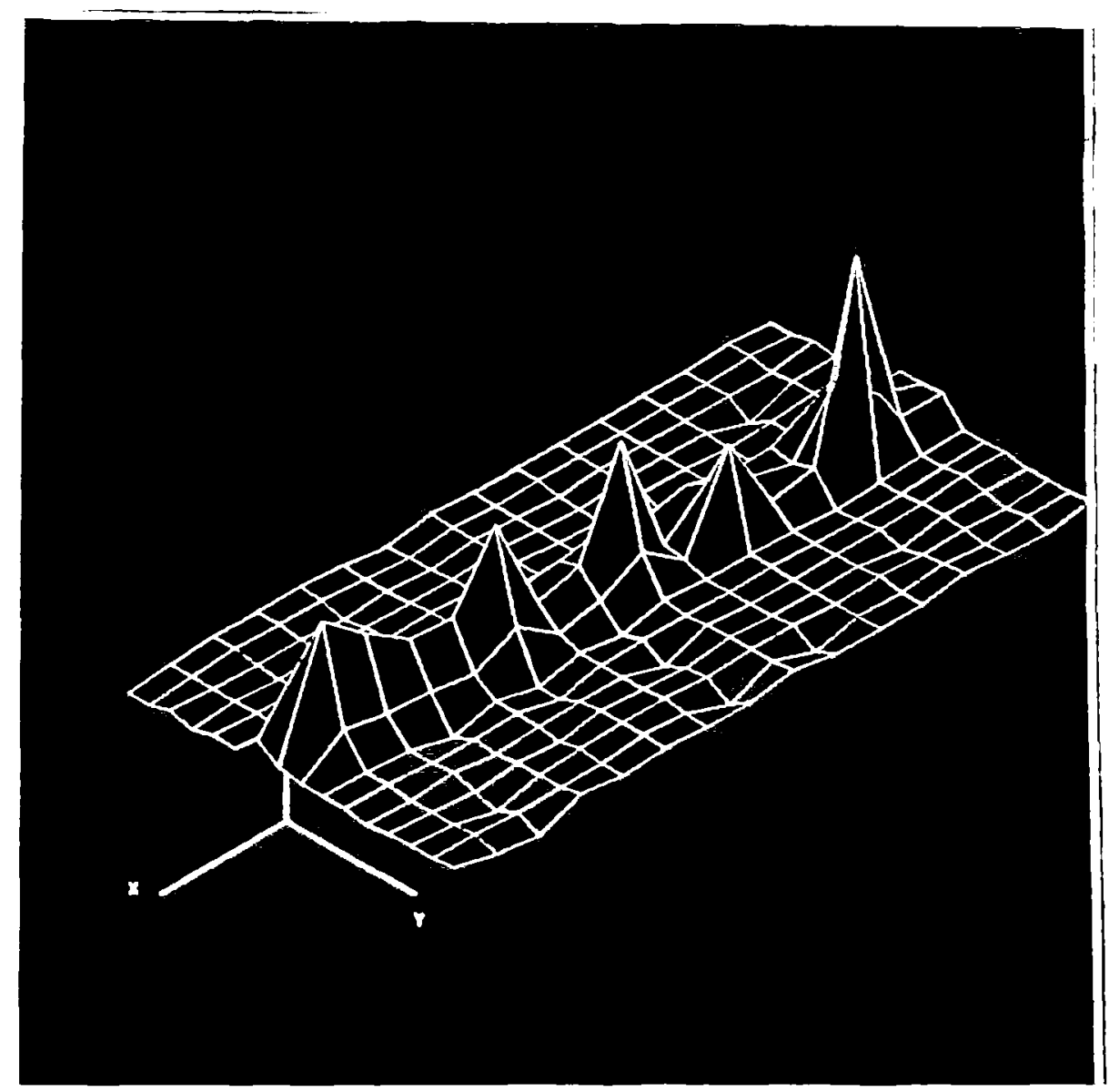

Figure 7: Filtered tactile responses for a linear edge. 


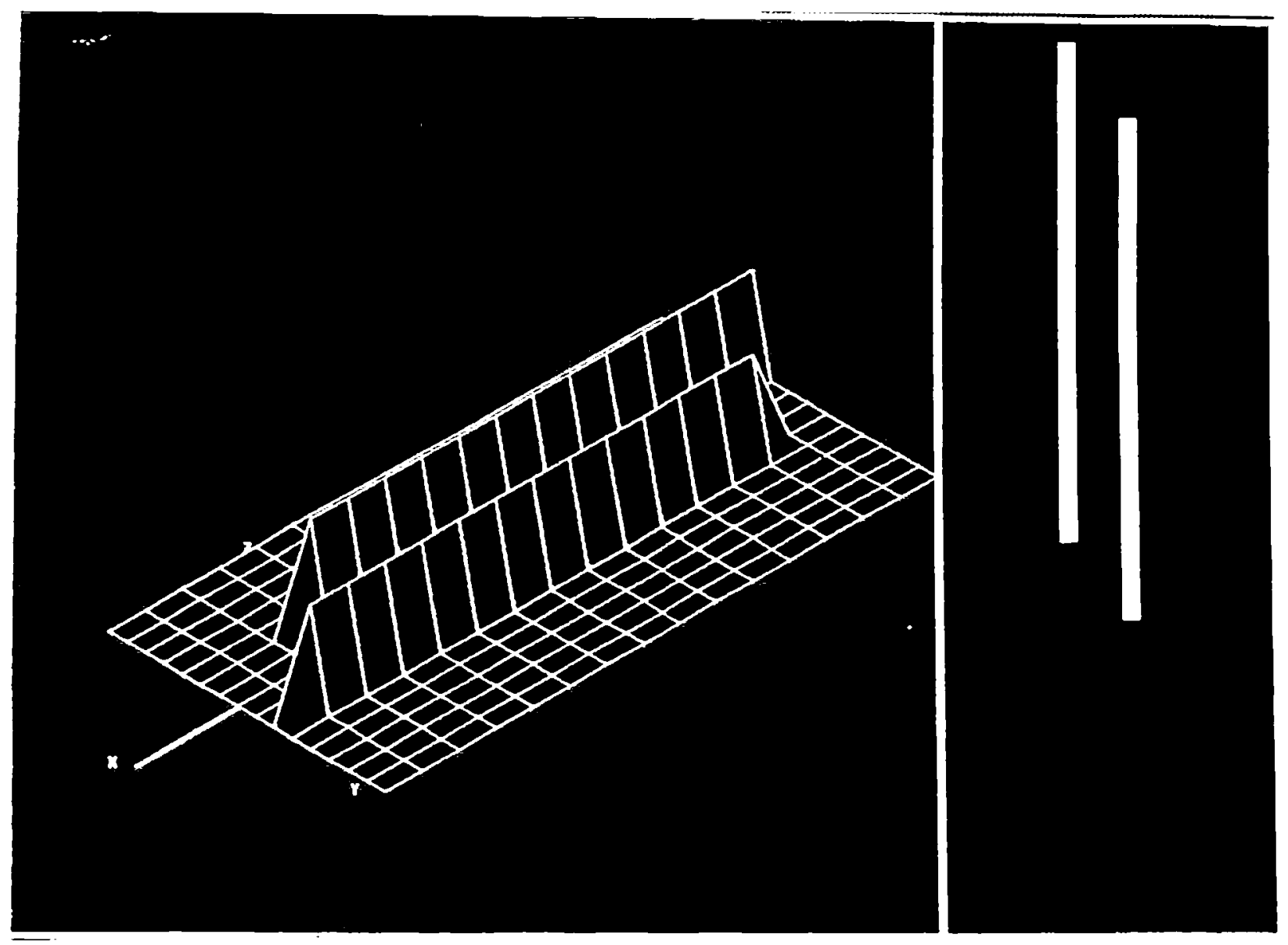

Figure 8: Extracted linear segments from Hough transform. 


\section{References}

1. Allen, Peter, "Sensing and describing 3-D structure," Proc. IEEE Conference on Robotics and Automation, pp. 126-131, San Francisco, April 7-10, 1986.

2. Allen, Peter K., Robotic object recognition using vision and touch, Kluwer Academic Publishers, Boston, 1987.

3. Bajcsy, Ruzena and Franc Solina, "Three dimensional object representation revisited," Proceedings International Conference on Computer Vision, London, June 1987.

4. Ballard, D. and C. Brown, Computer vision, Prentice-Hall, 1982.

5. Barr, Alan, "Superquadrics and angle preserving transformations," IEEE Computer Graphics and Applications, vol. 1, pp. 11-23, 1981.

6. Biederman, Irving, "Human image understanding: Recent research and a theory," Computer Vision. Graphics, and Image Processing, vol. 32, pp. 29-73, 1985.

7. Boult, T. and A. Gross, "Recovery of superquadrics from depth information," Proceedings of the AAAl Workshop on Spatial-Reasoning and Multisensor Integration, Morgan Kauffman, Los Altos. CA, Oct. 1987.

8. Briot, M., M. Renaud, and Z. Stojilkovic. "An approach to spatial pattern recognition of solid objects," IEEE Systems, Man, and Cybernetics, vol. SMC-8, pp. 690-694, 1978.

9. Brooks, Rodney, "Symbolic reasoning among 3-D models and 2-D images," Artificial Intelligence, vol. 17, pp. 285-349, 1981.

10. Cutkosky, Mark and Paul Wright. "Modeling manufacturing grips and correlation with the design of robotic hands," Proc. IEEE International Conference on Robotics and Automation, pp. 1533-1539. San Francisco, April 1986.

11. Ellis, R., Edward Riseman, and A. R. Hanson, "Tactile recognition by probing: Identifying a polygon on a plane," Proceedings of AAAI-86, pp. 632-637, Philadelphia, August 11-15, 1986.

12. Fearing, Ronald, "Simplified grasping and manipulation with dextrous robot hands," IEEE Journal of Robotics and Automation, vol. RA-2(4), pp. 188-195.

13. Grimson, W. E. L. and Tomas Lozano-Perez, "Model based recognition and localization from sparse three dimensional sensory data," A.I. memo 738, M.I.T. A.I. Laboratory, Cambridge, August 1983.

14. Gross, A. and T. Boult, "Error of fit measures for recovering parametric solids," International Conference on Computer Vision . Tarpon Springs, FL, December 1988.

15. Iberall, Thea, "The nature of human prehension: three dextrous hands in one," Proc. IEEE International Conference on Robotics And Automation. pp. 396-401. Raleigh. N. C., March 31-April 31987.

16. Interlink., The force sensing resistor: A new tool in sensor technology, $535 \mathrm{E}$. Montecito, Santa Barbara, CA, June 25, 1986.

17. Jacobsen, S. C., E. K. Iversen, D. F. Knutti, R. T. Johnson, and K. B. Biggers, "Design of the Utah/MIT dextrous hand," Proceedings of the IEEE Conference on Robotics and Automation, pp. 1520-1532, San Francisco, April 7-10, 1986.

18. Kobayashi, H., "Control and geometrical considerations for an ariculated robot hand," The International Journal of Robotics Research, vol. 4, no. 1, 1985. 
19. Lederman, Susan and Roberta Klatzky, "Hand movements: A window into haptic object recognition," Cognitive Science Technical Refort 8606, Univ. of California, Santa Barbara.

20. Lederman, Susan and Roberta Klatzky, "The intelligent hand," in The psychology of learning and motivation, ed. G. Bower, Academic Press.

21. Lyons, Damian, "A simple set of grasps for a dextrous hand," Proc. IEEE International Conference on Robotics And Automation, pp. 588-593, St. Louis, March 1985.

22. Marik, V., "Algorithms of the complex tactile information processing," Int. Joint Conf. Artificial Intelligence, pp. 773-774, 1981.

23. Marr, David, Vision, W. Freeman, San Francisco, 1982.

24. Mason, Matthew T. and J. Kenneth Salisbury, Robot hands and the mechanics of manipulation, M. I. T. Press, Cambridge, 1985.

25. Narasimhan, Sundar, David M. Siegel, John M. Hollerbach, Klaus Biggers, and George E. Gerpheide, "Implementation of control methodologies on the computational architecture for the Utah/MIT hand," Proc. IEEE International Conference on Robotics and Automation, pp. 1884-1889, San Francisco, April 7-10, 1986.

26. Pentland, Alex P., "Recognition by parts," Technical Report 406, SRI Intemational, December 16, 1986.

27. Solina, Franc, "Shape recovery and segmentation with deformable part models," Ph.D Dissertation, Deparment of Computer Science, University of Pennsylvania, December 1987.

28. Stansfield, Sharon, "Visually-guided haptic object recognition," Ph.D. dissertation, Department of Computer and Information Science, University of Pennsylvania, October 1987.

29. Stojilkovic, Z. and D. Saletic, "Leaming to recognize pattems by Belgrade hand prosthesis," Proc. 5th ISIR, pp. 407-413, 1975.

30. Tise, B., "A compact high resolution piezo-resistive digital tactile sensor," IEEE Conference on Robotics and Automation, pp. 760-764, Philadelphia, April 24-29, 1988.

31. Tomovic, Rajko, George Bekey, and Walter Kaplus, "A strategy for grasp synthesis with multi-fingered robot hands," Proc. IEEE International Conference on Robotics And Automation, pp. 83-89, Raleigh. N. C., March 31-April 31987. 